\title{
Luc Fraisse, Éric Wessler (dir.), L'écrivain et ses doubles
}

\section{Roberta Sapino}

\section{(2) OpenEdition}

\section{Journals}

Edizione digitale

URL: http://journals.openedition.org/studifrancesi/1010

DOI: $10.4000 /$ studifrancesi. 1010

ISSN: 2421-5856

Editore

Rosenberg \& Sellier

\section{Edizione cartacea}

Data di pubblicazione: 1 agosto 2015

Paginazione: 430-432

ISSN: 0039-2944

Notizia bibliografica digitale

Roberta Sapino, «Luc Fraisse, Éric Wessler (dir.), L'écrivain et ses doubles », Studi Francesi [Online], 176 (LIX | II) | 2015, online dal 01 août 2015, consultato il 18 septembre 2020. URL : http:// journals.openedition.org/studifrancesi/1010; DOI : https://doi.org/10.4000/studifrancesi.1010

Questo documento è stato generato automaticamente il 18 settembre 2020.

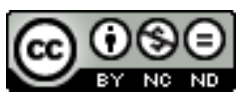

Studi Francesi è distribuita con Licenza Creative Commons Attribuzione - Non commerciale - Non opere derivate 4.0 Internazionale. 


\title{
Luc Fraisse, Éric Wessler (dir.), L'écrivain et ses doubles
}

\author{
Roberta Sapino
}

\section{NOTIZIA}

LUC FRAISSE, ÉRIC WESSLER (dir.), L'écrivain et ses doubles, Paris, Classiques Garnier, 2014, pp. 542 .

1 Il volume, che si presenta come una «enquête sur le personnage autoréflexif dans la littérature européenne» (seppur l'area francofona prevalga), si articola in tre sezioni nelle quali il personaggio autoriflessivo è analizzato nei suoi rapporti con l'identità autoriale, con l'elaborazione di un'ars poetica e con l'affermazione di uno specifico sguardo sulla letteratura.

2 A preparare il terreno, un importante apparato introduttivo ad opera dei due curatori. Partendo dall'assunto che il bisogno di dotarsi di un doppio di sé - di un'ombra che rassicuri sulla propria esistenza e venga in aiuto di un'identità incerta e minacciata dall'incombere della morte - sia prerogativa di qualsiasi essere umano, almeno nelle culture occidentali, e che a caratterizzare lo scrittore sia la necessità di perpetrare tale ricerca da solo, nello spazio della propria scrittura, Éric WESSLER (Introduction. Le double de l'écrivain comme fondement de la littérature, pp. 7-23) mette in rapporto l'affermarsi progressivo di forme di scrittura autoriflessive con il consolidarsi di un'idea di letteratura autonoma da manifestazioni esterne-religiose, politiche...-e con il conseguente emergere dell'autore come «créateur absolu» capace di trasformare la propria soggettività in opera. Luc FRAISSE (Envoi. L'interprétation du personnage autoréflexif chez les théoriciens du roman, pp. 25-39) prende poi la parola per tracciare un agile percorso tra il pensiero di alcuni dei principali teorici del romanzo (Stanesco, Robert, Girard, Goldmann, Bakhtin, Kundera) che si sono interrogati sull'autoriflessività del personaggio in quanto manifestazione simbolica sia di una concezione estetica generale, sia del processo creativo di un dato testo. 
3 La prima parte, intitolata "Les dédoublements de l'écrivain et la quête de l'identité», si apre con Gérard GROS (Le poète et ses personnages. Étude sur l'art des dédoublements chez Villon, pp. 43-60), che si addentra tra le affermazioni e contraddizioni biografiche che fanno dell'opera di Villon un testamento giocoso in cui il gusto per la messa in scena e per la moltiplicazione delle voci contribuisce a mascherare più che a rivelare l'io del poeta. Olivier LARIZZA (Mary Shelley et ses doubles. Le personnage autoréflexif dans Frankenstein, pp. 61-83) afferma che la discrezione mantenuta da Mary Shelley nella vita sarebbe compensata da una forte esposizione di sé nella scrittura: parallelamente alla vicenda del mostro, si può leggere quella di un'autrice che dissimula nei tre protagonisti - maschili - la sua ambizione di compiere l'impresa prometeica. In George Borrow et ses doubles (pp.85-100), Maxime LEROY si sofferma su Lavengro, opera annunciata come autobiografia ma nella quale finzione e intertestualità intervengono cospicuamente nel tessuto della memoria, originando un complesso sistema di personaggi autoriflessivi in cui l'autore, anticipando Freud, scinde in una costellazione di «moi partiels» le diverse correnti della sua soggettività. Lamentando come gli studi sull'autoriflessività letteraria tendano a rivolgersi a un corpus ristretto di opere esemplari, Michèle TOURET (De deux romans cendrarsiens. Ou le personnage (auto)réflexif où on ne l'attendrait pas, pp. 101-115) si rifà alle riflessioni di J-M. Schaeffer e dimostra come il personaggio riflessivo costituisca la chiave di volta sia della creazione sia della comprensione di romanzi come Moravagine e Dan Yack. Il contributo successivo, di Volker RoLoff (Autoréflexion, autofiction et automédialité. Les doubles chez Robbe-Grillet, pp. 117-131), ritorna invece su un autore topico come Alain Robbe-Grillet, proponendo di sostituire la nozione di autobiografia con quella di «automédialité», più adatta a una riflessione sul doppio coerente e multiforme, che si concretizza non solo nella scrittura ma anche nei film, nei ciné-romans e nei picto-romans. La creazione di personaggi di scrittori alle prese con un qualche genere di fallimento è una delle cifre dell'opera di Imre Kertész, osserva Frédérique TOUDOIRE-SURLAPIERRE (Liquider ses dettes? Imre Kertész et ses doubles, pp. 133-148), e consente all'autore di interrogarsi sulla propria identità di "écrivain hongrois», problematicamente situata tra quella di uomo ungherese deportato ad Auschwitz e quella di scrittore tout court. Ralph HEYNDELS (Abdellah à jamais disparu ou les jeux de miroir du je. Émergence et évanescence de soi dans la mise en scène de l'écriture chez Abdellah Taia, pp.149-161) mette in luce le commistioni talvolta paradossali tra finzione della realtà e realtà della finzione generate nell'opera di Taia, segni di un io scrivente che esita a riconoscersi nell'io di carta. Più vasto è il campo coperto dal contributo di Yves-Michel ERGAL (L'auteur e(s)t moi, pp.163-171) che, partendo da un romanzo recente di Éric Chevillard, propone una panoramica delle questioni sollevate da alcuni autori-cardine della scrittura autoriflessiva come Beckett, Sterne, Dickens, Proust e Cervantes. Chiude la sezione Stéphane CHAUDIER, con un articolo dal tono brillante che, come rispondendo all'appello di TOURET a rivolgersi a testi meno frequentati, si serve di alcune teorie di Leo Strauss e Clément Rosset per ricercare l'immagine di sé che Échenoz in 14 lascia trapelare non tanto da un personaggio, quanto piuttosto dallo stile (L'écrivain, son style et son double dans "14" de Jean Échenoz, pp. 173-195).

4 José Manuel LOSADA GOYA (L'accession d'Hugo au statut de mage, pp. 199-215) inaugura la sezione intitolata «Le personnage autoréflexif, incarnation de l'art de l'écrivain» con un intervento che ripercorre sinteticamente l'evoluzione dell'istanza poetica presente in Odes et Ballades, Les Orientales, Les Châtiments e Les Contemplations, mostrando i legami 
complessi che essa instaura con la biografia dell'uomo Victor Hugo. In L'artiste et ses doubles mythiques dans "Moralités Légendaires" de Laforgue (pp. 217-237), Patrick LABARTHE legge il pluralizzarsi della soggettività di Laforgue in una costellazione di doppi mitici come una riflessione storicamente situata sulla demistificazione dello statuto d'artista, mentre David ELLISON (Le personnage autoréflexif et le lieu spéculaire. Les «Venises» de Marcel Proust et de Thomas Mann, pp. 239-257) mette in luce una materia comune, tanto etica quanto estetica, presente nelle rappresentazioni di Venezia ad opera di Proust e Mann: per entrambi l'inscrizione letteraria della città risulta nella creazione di un lieu spéculaire che riflette il dramma del personaggio autoriflessivo, richiamando l' Unheimlichkeit freudiana. Sjef HOUPPERMANS (De doublure en doublure. Raymond Roussel écrivain, pp. 259-271) analizza alcuni tra i doppi autoriflessivi generati dalla penna dello scrittore, colto non solo nella sua attività di creatore, ma anche nel suo continuo oscillare tra ybris e il timore del fallimento; Jean-François LOUETTE (Le peintre et l'écrivain dans "Les Mémoires de Dirk Raspe", pp. 273- 289) si concentra invece sull'ultimo romanzo di Drieu la Rochelle e in particolare sulle figure femminili, che egli legge come portatrici di diverse possibilità estetiche: l'opera si rivela un mezzo per lo scrittore per meditare sulla sua arte: i rapporti tra le differenti beautés e il protagonista Dirk Raspe, doppio di Van Gogh, costituirebbero allora un'ars poetica diffusa. A seguire, L'Art de Bosco, thème romanesque? (pp. 291-303) mostra come le aspirazioni spiritualiste dell'arte di Henri Bosco si traducano in quello che Michel ARouimi definisce un mandala testuale in cui la soggettività dell'autore è disciolta non solo nella rete dei personaggi, ma anche nell'ambiente che li accoglie. In Lire le monde avec le personnage perecquien (pp. 305-323), Steen Bille JøRGENSEN, dopo aver sottolineato la necessità di guardare all'autoriflessività non solo dal punto di vista dello scrittore ma anche di quello del fruitore del testo, osserva che in La vie mode d'emploi i progetti individuali dei diversi personaggi riflettono il progetto letterario di Perec. Comment Ionesco se met en scène en tant qu'écrivain (pp. 325-341) di Marie-Claude HUBERT muove dalla constatazione che le pièces di Ionesco siano straordinariamente ricche di quei «mots d'auteur» con cui il drammaturgo, conscio della sua responsabilità di testimone del suo tempo, presta ai personaggi idee $\mathrm{e}$ parole sull'arte e sulla vita. Éric WESSLER ("Le promeneur solitaire" de Beckett. Ou les rêveries de l'écrivain moderne, pp.343-365), infine, considera che se la beckettiana strada di campagna può essere letta come una metafora autoriflessiva della scrittura, allora nei personaggi in marcia - fattuale o ipotizzata - è lecito vedere un doppio dello scrittore, combattuto tra avanzamento e immobilità, desiderio e sfiducia nella possibilità di dire.

5 La terza e ultima sezione, "La Littérature au miroir», si apre con un balzo nel tempo fino all'epoca medievale: in Merlin, le double du double du romancier. Lecture du "Roman du Graal" de Robert de Boron (pp. 369-380), Jan HERMAN rintraccia le caratteristiche che fanno del personaggio di Merlino il portatore di due posture del romanziere rispetto a Dio creatore supremo. Paul PELCKMANS (Doublures inutiles. Les personnages romanciers d'Isabelle de Charrière, pp. 381-393) si avventura nell'opera ancora relativamente poco studiata di Isabelle de Charrière, lettrice instancabile ma autrice più scostante, per constatare lo scarso spessore dei tre personaggi di romanzieri che vi compaiono, segno forse di un celato scetticismo verso l'utilità delle lettere. In Le personnage autoréflexif dans l'espace autobiographique. L'exemple de Gide (pp. 395-405), Jean-Michel WITTMANN si concentra sui personaggi secondari della prima parte di Si le grain ne meurt, invitando a dimenticare le questioni di referenzialità per leggervi invece un sistema centripeto di rilessi che problematizza ancora una volta la concezione della scrittura e della lettura; Audrey 
BLIND (Le personnage de l'Irrépressible dans "Le Soulier de satin" de Paul Claudel, pp. 407-416) presenta poi la figura dell'Irrepressible come un doppio della concezione claudeliana dell'opera teatrale, mentre Julien LINGENSER (L'écrivain-réalisateur et ses doubles chez Mario Soldati, pp.417-436) analizza 24 ore in uno studio cinematografico, Le lettere da Capri et L'attore, realizzando un bel ritratto di un artista che scelse di essere principalmente uomo di lettere nella vita e personaggio cinematografico nella letteratura. Il contributo di Luc FRAISSE (Quand le personnel romanesque incarne une conception du roman. "Les Gommes" d'Alain Robbe-Grillet, pp. 437-477) osserva che in Les Gommes il messaggio autoriflessivo, tratto tipico del Nouveau Roman, manifesta l'eccezionalità di essere portato dall'insieme dei personaggi: contrastando la possibilità di un narratore onnisciente, essi sollevano l'interrogazione sul senso nell'opera letteraria.

6 Mechthild ALBERT (Le personnage autoréflexif dans le roman métahistorique espagnol, pp. 479-494), si concentra su Beatus Ille di Antonio Muñoz Molina e ¡Otra maldita novela sobre la guerra civil! di Isaac Rosa per discutere i tratti peculiari e i limiti di quella letteratura nata in epoca postmoderna e dedicata a recuperare la memoria della Guerra civile e della successiva dittatura, nella quale la ricerca storica e la riflessione sulla narrazione si traducono nella forma del quest plot. Peter SCHNYDER («Il ne s'agit pas d'un exercice de poésie». Poésie et autoréflexivité chez Philippe Jaccottet, pp. 495-511) dimostra, anche numericamente, l'importanza dell'autoriflessività all'interno dell'opera di Jaccottet, e il suo ruolo di «passerelle» tra la riflessione e l'affermazione delicata, che non preclude mai un'interrogazione ulteriore. L'ultimo articolo della raccolta è Miroitements de l'écrivain dans "Atonement" de Ian McEwan. Le roman à l'épreuve de l'autoréflexivité (pp.513-525) di Charline PLUVINET, in cui la coesistenza di più figure autoriali e di più racconti possibili è interpretata come il segno di un desiderio di far fronte alle aporie del romanzo ricercando una nuova relazione tra esso e il reale. 